\title{
The Role of Akt2 and CA-125 Serum Levels as Predictors for Successful Cytoreduction in Epithelial Ovarian Cancer Surgery
}

\author{
Yudi Mulyana Hidayat ${ }^{1}$, Gatot Nyarumenteng Adhipurnawan Winarno ${ }^{1}$, \\ Maringan Diapari Lumban Tobing ${ }^{1}$, Arieff Kustiandi ${ }^{1}$, Kemala Isnainiasih Mantilidewi ${ }^{1}$, \\ Sofie Rifayani Krisnadi ${ }^{1}$ \\ ${ }^{1}$ Department of Obstetrics and Gynecology, Faculty of Medicine, Universitas Padjadjaran-Dr Hasan Sadikin General Hospital, Jl. Pasteur \\ No. 38, Bandung 40161, West Java, Indonesia
}

\begin{abstract}
Summary
Ovarian cancer is one of the common causes of cancer deaths in gynecological malignancies in Indonesia, and indeed the world. Of all classifications of ovarian cancer, the epithelial subtype shows the highest incidence. Cytoreductive surgery is a determinant of prognosis for patients with advanced epithelial ovarian cancer. One possible way to predict the results of cytoreductive surgery is to measure preoperative tumor marker levels. This study aimed to assess the validity of serum levels of Protein kinase $\mathrm{B} \beta$ (Akt2) and cancer antigen-125 (CA-125) as outcome predictors for cytoreduction in epithelial ovarian cancer surgery. This was an observational, analytical, cross-sectional study. Akt2 and CA-125 serum levels were examined by enzyme-linked immunosorbent assay (ELISA) and immunoassay technique, respectively. Mean levels of Akt2 and CA-125 were significantly different between optimal and suboptimal cytoreduction groups $(p<0.05)$. The cut-off point (COP) value obtained for Akt 2 was $1.20 \mathrm{U} / \mathrm{mL}$ with area under curve (AUC) value of $68.9 \%$, while the COP for CA-125 was $222.50 \mathrm{U} / \mathrm{mL}$ with AUC value of $75.3 \%(p<0.05)$. If the preoperative Akt2 serum level was greater than $1.20 \mathrm{U} / \mathrm{mL}$, and preoperative CA-125 level was greater than $222.5 \mathrm{U} / \mathrm{mL}$, the probability of suboptimal cytoreduction was increased. Thus, our study highlights the possibility for Akt2 and CA-125 levels to be used as serum biomarkers in predicting the success of cytoreductive surgery.
\end{abstract}

Key words: Epithelial ovarian cancer; cytoreduction; Akt2; CA-125.

\section{Introduction}

Ovarian cancer is one of the leading causes of death from cancer in women worldwide. In 2012, there were almost 240,000 new cases of ovarian cancer, ranking it the seventh highest of all cancers in women. By 2018, this figure had risen to almost 300,000, making it the fourth most common cancer in women. Ovarian cancer was the cause of death in 151,917 cases and 184,799 cases worldwide in 2012 and 2018 , respectively $[1,2]$.

Classification of malignant ovarian cancer consists of epithelial-, stromal sex-cordal and germ cell-type; 90\% of malignant ovarian cancers are of the epithelial type [3, 4].The standard management of epithelial ovarian cancer is surgical staging in early-stage (stage 1) cases and cytoreductive surgery in cases of advanced stage (stages 2, 3 and 4), followed by platinum-taxane based chemotherapy [5].

Cytoreductive surgery is performed on advanced stage epithelial ovarian cancer to remove as much of the tumor bulk as possible. Intraoperatively by gross examination, surgery is considered as optimal cytoreduction if residual tumor $<1 \mathrm{~cm}$ at greatest dimension, while cytoreduction is suboptimal if the residual tumor is $>1 \mathrm{~cm}[3,5,6]$. Saitou et al. reported that patient prognosis only improved when residual tumor nodules were $<1 \mathrm{~cm} \mathrm{[7].}$
Measurement of tumor marker levels is among the outcome predictors of cytoreductive surgery. Cancer antigen125 (CA125) serum level $>420 \mathrm{U} / \mathrm{ml}$ was a strong predictor of suboptimal cytoreduction surgery in patients with epithelial ovarian cancer [8].The combination of CA-125 levels with other tumor markers may help determine management [9]. The increase in CA-125 causes an increase in Protein kinase B(AKT) expression [10]. AKT is a key signaling molecule that regulates cellular processes such as proliferation, cell growth and metabolism as well as serving as a tumor marker [11]. Of the three AKT isoforms, AKT2 expression is found elevated in ovarian cancer [12]. Overexpression of AKT2 leads to increased expression of pyruvate kinase (PKM2) in ovarian cancer cells in nude mice [13]. Elevation of AKT2 expression induced cell migration and invasion in vitro, as well as lung metastasis in vivo, while suppression of AKT2 blocked these effects. STAT3 expression was elevated and NF- $\kappa \mathrm{B}$ p 65 nuclear translocation was activated both in vitro and in vivo when AKT2 was overexpressed; these effects were inhibited when AKT2 expression was suppressed. AKT2 increases the migration, progression and invasion of ovarian cancer cells in vitro and in nude mice in vivo through PKM2-mediated elevation of STAT3 expression and NF- $\kappa$ B activation [13].
Eur. J. Gynaecol. Oncol. - ISSN: 0392-2936 XLI, n. 5, 2020

doi: $10.31083 /$ j.ejgo.2020.05.2116
This is an open access article under the CC BY-NC 4.0 license (https://creativecommons.org/licenses/by-nc/4.0/). 
Preoperative combination of AKT2 and CA-125 levels may predict the outcome of cytoreductive surgery, which in turn may help clinicians determine the subsequent management of patients with advanced ovarian cancer.

\section{Methods}

This study was registered and approved by the Research Ethics Committee, Faculty of Medicine Padjadjaran University/Dr. Hasan Sadikin Hospital, Bandung, Indonesia, under trial registration number 36/UN6.KEP/EC/2018 on April 13 ${ }^{\text {th }}, 2018$.

\section{Study subjects}

This study was a prospective, observational analysis with a cross-sectional design. The population included patients with a diagnosis of suspected advanced ovarian cancer who would undergo cytoreductive surgery at Dr. Hasan Sadikin General Hospital, Bandung. The inclusion criteria were new patients with suspected epithelial ovarian cancer undergoing cytoreductive surgery who were willing to give informed consent, and who were not suffering from chronic diseases or other tumors. Consecutive patients from the 2018-2019 period attending Dr. Hasan Sadikin General Hospital, Bandung were included.

Before surgery, blood samples were taken from the entire study population for the assessment of serum biomarker levels. Patients who were confirmed histopathologically to not suffer from epithelial ovarian cancer, or who only had stage I disease, were excluded from the study. Patients were also excluded if their histopathological preparations were damaged or could not be assessed or if they were not willing to provide informed consent.

\section{Materials}

AKT2 serum examination was performed by ELISA (Enzyme-Linked Immunosorbent Assay. Cloud-Clone Corp., USA). CA-125 examination was done by immunoassay technique analyzed by ADVIA Centaur ${ }^{\circledR}$ XP system (Siemens Healthcare Diagnostics Inc., USA). All techniques were done according to the manufacturer's instructions.

\section{Statistical Analysis}

All data were assessed statistically using $\operatorname{SPSS} \AA$ (24.0.0). For normally distributed data, a paired $t$-test analysis was used, while a Mann Whitney analysis was used for not normally distributed data. As for categorical data, $p$-values were analyzed using the Chi-Square test; if the Chi-Square requirements were not met, then Kolmogorov Smirnov and Exact Fisher tests were used. The cut-off point (COP) was made using receiver operating characteristic (ROC) curve 23 analysis.

\section{Results}

Fifty-three patients were included in this study, divided into suboptimal and optimal cytoreductive groups. Char- acteristics of the subjects in the two groups were analyzed. There were no significant differences $(p>0.05)$ between the two groups regarding patients' age, parity, height, weight, body mass index, ascites, bleeding, staging, and histopathology (Table 1). Analysis of blood samples taken before surgery revealed the levels of serum AKT2 and CA125 had a significant difference $(p<0.05)$ between that of suboptimal and optimal cytoreductive groups (Table 2).

The COP of AKT2 was $1.20 \mathrm{U} / \mathrm{mL}$, with a significant $\mathrm{p}$ value $(p<0.05)$ between COP and results of cytoreduction (Table 3). The AUC value of AKT2 levels with ROC method was $68.9 \%$, implying that $68.9 \%$ of patients with AKT2 serum levels greater than $1.20 \mathrm{U} / \mathrm{mL}$ would undergo suboptimal cytoreduction with $76.2 \%$ specificity, $65.6 \%$ sensitivity, and $69.8 \%$ accuracy (Figure 1, $p=0.021$ ).

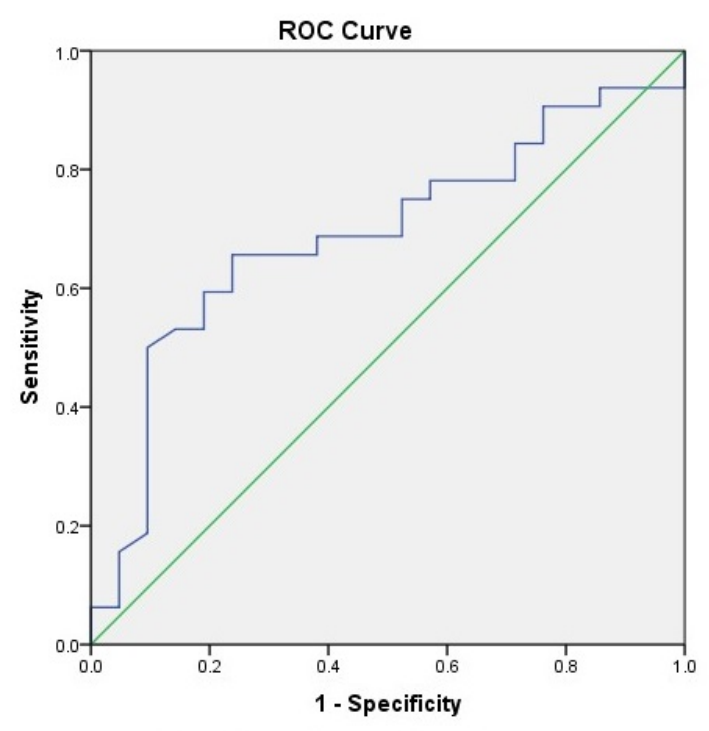

Diagonal segments are produced by ties.

Figure 1. - Akt2 with Cytoreduction. The AUC value obtained from the ROC method was $68.9 \%$ ( $p=0.021)$, meaning that Akt2 can predict cytoreduction correctly in 37 patients out of a total of 53 patients.

The COP of CA-125 obtained from this study was 222.5 $\mathrm{U} / \mathrm{mL}$, with a significant $\mathrm{p}$ value $(p<0.05)$ between COP and results of cytoreduction (Table 4 ). Based on the ROC method, the AUC value received was $77.5 \%$, suggesting that $77.5 \%$ of patients with serum CA-125 levels exceeded $222.5 \mathrm{U} / \mathrm{ml}$. Suboptimal cytoreduction was predicted with $61.9 \%$ specificity, $75 \%$ sensitivity and $69.8 \%$ accuracy (F igure $2, p=0.002$ ).

Multivariate analysis was obtained by logistic regression test to determine whether AKT2 or CA-125 was more closely related to the cytoreduction result. As Table 5 shows, AKT2 had a comparably smaller $\mathrm{p}$ value than CA125 ( $p=0.003$ vs. $p=0.005$, respectively), and higher odds ratio $(\mathrm{OR}=12.404 v s . \mathrm{OR}=10.379$, respectively). These data indicate that AKT2 measurement may be more likely 
Table 1. - Comparison of Characteristics of Patients in Optimal and Suboptimal Cytoreduction groups.

\begin{tabular}{|c|c|c|c|}
\hline \multirow{2}{*}{ Variable } & \multicolumn{2}{|c|}{ Group } & \multirow{2}{*}{$p$ Value } \\
\hline & Suboptimal cytoreduction $n=32$ & Optimal cytoreduction $n=21$ & \\
\hline Age (years) & & & 0.131 \\
\hline Mean \pm SD & $50.09 \pm 9.686$ & $45.23 \pm 13.374$ & \\
\hline Parity & & & 0.979 \\
\hline 0 & $7(21.9 \%)$ & $2(9.5 \%)$ & \\
\hline 1 & $4(12.5 \%)$ & $8(38.1 \%)$ & \\
\hline 2 & $8(25.0 \%)$ & $1(4.8 \%)$ & \\
\hline$>3$ & $13(40.6 \%)$ & $10(47.6 \%)$ & \\
\hline Height (cm) & & & 0.164 \\
\hline Mean $\pm \mathrm{SD}$ & $153.09 \pm 5.348$ & $150.80 \pm 4.285$ & \\
\hline Weight Preop (kg) & & & 0.056 \\
\hline Mean $\pm \mathrm{SD}$ & $56.25 \pm 8.925$ & $51.19 \pm 9.825$ & \\
\hline Weight Postop & & & 0.057 \\
\hline Mean $\pm \mathrm{SD}$ & $50.12 \pm 9.317$ & $45.26 \pm 8.169$ & \\
\hline BMI & & & 0.175 \\
\hline Mean \pm SD & $21.44 \pm 4.259$ & $19.90 \pm 3.501$ & \\
\hline Ascites & & & 0.195 \\
\hline Mean \pm SD & $1892.50 \pm 3126.565$ & $2281.90 \pm 5117.13$ & \\
\hline Bleeding Volume & & & 0.114 \\
\hline Mean \pm SD & $1312.10 \pm 920.868$ & $1357.14 \pm 1826.08$ & \\
\hline Stage & & & 0.149 \\
\hline II & $5(15.6 \%)$ & $10(47.6 \%)$ & \\
\hline III & $20(62.5 \%)$ & $11(52.4 \%)$ & \\
\hline IV & $7(21.9 \%)$ & $0(0.0 \%)$ & \\
\hline Histopathology & & & 0.873 \\
\hline Serous & $13(40.6 \%)$ & $6(28.6 \%)$ & \\
\hline Mucinous & $7(21.9 \%)$ & $10(47.6 \%)$ & \\
\hline Endometrioid & $7(21.9 \%)$ & $2(9.5 \%)$ & \\
\hline Clear Cell & $5(15.6 \%)$ & $3(14.3 \%)$ & \\
\hline
\end{tabular}

Age, Weight PostOp (preoperative), and BMI were analyzed by Independent t-Test. Height, Weight PreOp (preoperative), Ascites, and Bleeding Volume were analyzed by Mann Whitney test. Parity, Stage, and Histopathology were analyzed by Kolmogorov Smirnov test.

Table 2. - Comparison of Akt2 and CA-125 in the Suboptimal and Optimal Cytoreduction groups.

\begin{tabular}{|c|c|c|c|}
\hline \multirow[b]{2}{*}{ Variable } & \multicolumn{2}{|c|}{ Groups } & \multirow[b]{2}{*}{$p$ Valuc } \\
\hline & Suboptimal cytoreduction $n=32$ & Optimal cytoreduction $n=21$ & \\
\hline Akt2 & & & $0.021 *$ \\
\hline Mean $\pm \mathrm{SD}$ & $1.41 \pm 0.555$ & $1.12 \pm 0.286$ & \\
\hline Median & 1.43 & 1.09 & \\
\hline Range & $0.65-3.79$ & $0.80-1.89$ & \\
\hline CA-125 & & & $0.002 *$ \\
\hline Mean $\pm \mathrm{SD}$ & $964.22 \pm 1722.532$ & $264.98 \pm 251.883$ & \\
\hline Median & 600.00 & 132.70 & \\
\hline Range & $4.29-9934.00$ & $5.10-701.00$ & \\
\hline
\end{tabular}

to accurately predict optimal tumor resection probability.

Both AKT2 and CA-125 had $p$-value $<0.05$ according to multivariate analysis, (table 6). The new cut-off point was generated by re-coding AKT2 value based on the orig- inal AKT2 COP 1.20. AKT2 values $<1.20$ were re-coded to " 1 " and values $>1.20$ were re-coded to " 2 ". The recoded values were multiplied by corresponding CA-125 to produce the combined variable. An ROC curve was plotted 
Table 3. - Comparison between Cut-Off Points of Akt 2 in Suboptimal and Optimal Cytoreduction Groups.

\begin{tabular}{lccc}
\hline \multicolumn{4}{c}{ Cytoreduction } \\
\hline Variable & Suboptimal n $=\mathbf{3 2}$ & Optimal n $=\mathbf{2 1}$ & $\boldsymbol{p}$ Value \\
\hline Akt2 & & & $\mathbf{0 . 0 0 3 *}$ \\
$>1.20$ & $21(65.6 \%)$ & $5(23.8 \%)$ & \\
$<1.20$ & $11(34.4 \%)$ & $16(76.2 \%)$ & \\
\hline
\end{tabular}

${ }^{*} p<0.05$ (chi-square test).

Table 4. - Comparison between Cut-off Points of CA-125 in Suboptimal and Optimal.

\begin{tabular}{lccc}
\hline \multicolumn{4}{c}{ Cytoreduction } \\
\hline Variable & Suboptimal $\mathbf{n}=\mathbf{3 2}$ & Optimal $\mathbf{n}=\mathbf{2 1}$ & $\boldsymbol{p}$ Value \\
\hline Ca-125 & & & $\mathbf{0 . 0 0 7 *}$ \\
$>222.50$ & $24[75.0 \%]$ & $8[38.1 \%]$ & \\
$<222.50$ & $8[25.0 \%]$ & $13[61.9 \%]$ & \\
\hline
\end{tabular}

$* p<0.05$ (chi-square test).

from the combination variable, which resulted in the combination COP was $410.45 \mathrm{U} / \mathrm{mL}$. The new COP had a $75 \%$ sensitivity, $61.9 \%$ specificity and $69.8 \%$ accuracy for predicting optimal cytoreduction (Figure 3, $p=0.001$ ).

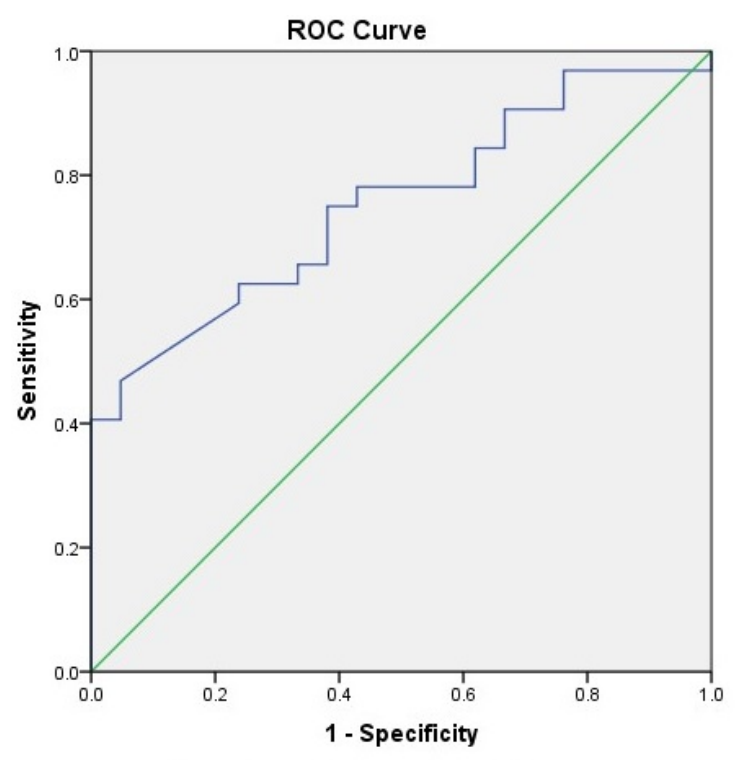

Diagonal segments are produced by ties.

Figure 2. - CA-125 with Cytoreduction. The AUC value obtained from the ROC method was $75.3 \%(p=0.002)$, meaning that CA- 125 can predict cytoreduction correctly in 40 patients out of a total of 53 patients.

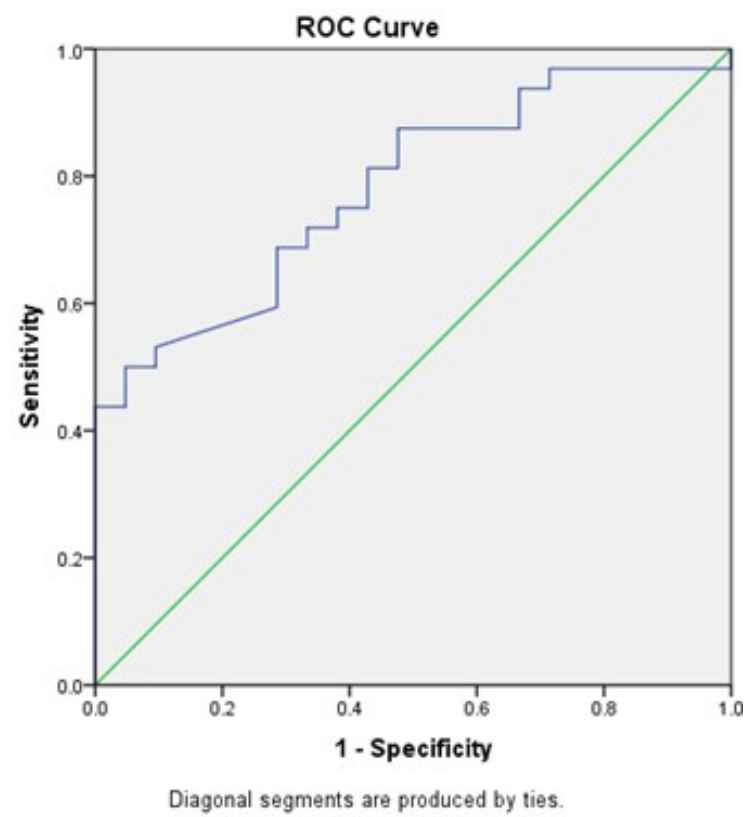

Figure 3. - The combination of Akt2 and CA-125 with Cytoreduction. The AUC value obtained from the ROC method was $78.1 \%(p=0.001)$, meaning that combination of Akt2 and CA125 can predict cytoreduction correctly in 41 patients out of 53 patients.

\section{Discussion}

Cytoreductive surgery in epithelial ovarian cancer patients determines prognosis and subsequent therapy. Studies have shown that patients with optimal cytoreduction have a better prognosis. Optimal cytoreduction with a residual mass $<1 \mathrm{~cm}$ increases the survival rate by 22 months [14].

The AKT signaling pathway is key in mediating the survival of tumor cells and avoiding apoptosis [13]. A 2014 meta-analysis conducted by Cai et al. that reviewed 11 articles stated that a high expression of AKT was related to poor survival in patients with epithelial ovarian cancer. The authors concluded that only high expression of AKT was significantly related to a poor prognosis, reflecting its oncogenic nature [15].

In this study, the average serum level of AKT2 was statistically significantly different between the optimal and suboptimal cytoreductive group. The COP of AKT2 was $1.20 \mathrm{U} / \mathrm{mL}$, with a significant $p$ value between COP and results of cytoreduction. The AUC value of AKT2 levels with ROC method was $68.9 \%$, implying that $68.9 \%$ of patients with AKT2 serum levels more than $1.20 \mathrm{U} / \mathrm{mL}$ would undergo suboptimal cytoreduction with $76.2 \%$ specificity, $65.6 \%$ sensitivity The results of this study indicate that an increase in AKT expression will make it more difficult to achieve optimal tumor cytoreduction. This is consistent with the theory that high AKT expression is associated with a worse prognosis since oncogenic activity in the Akt sig- 
Table 5. - Multivariate COP of Akt2 and CA-125 Analysis.

\begin{tabular}{|c|c|c|c|c|c|c|c|}
\hline & & $\mathrm{B}$ & S.E. & $p$ value & OR & Lower & $\begin{array}{c}\text { 95\% C.I.for OR } \\
\text { Upper }\end{array}$ \\
\hline \multirow[t]{2}{*}{ Step 1} & CutoffAkt2 & 2.518 & 0.846 & $0.003^{*}$ & 12.404 & 2.365 & 65.070 \\
\hline & CutoffCA-125 & 2.340 & 0.837 & $0.005^{*}$ & 10.379 & 2.014 & 53.487 \\
\hline
\end{tabular}

Cytoreduction Groups

Note: ${ }^{*} p<0.05$ (logistic regression test)

Table 6. - Comparison between Cut-off Points of CA-125 in Suboptimal and Optimal.

\begin{tabular}{lccc}
\hline \multicolumn{4}{c}{ Cytoreduction } \\
\hline Variable & Suboptimal $\mathrm{n}=32$ & Optimal $\mathrm{n}=21$ & $\boldsymbol{p}$ Value \\
\hline Combinations CA-125 dan Akt2 & & & $0.006^{*}$ \\
$>410.45$ & $23(71.9 \%)$ & $7(33.3 \%)$ & \\
$<410.45$ & $9(28.1 \%)$ & $14(66.7 \%)$ & \\
\hline
\end{tabular}

Note: ${ }^{*} p<0.05$ (chi-square test)

naling pathway is known to have an essential role in tumorigenesis by influencing the absorption of glucose, amino acids, and lipids [11]. Thus, AKT2 serum levels can be used to predict whether results of cytoreduction will be optimal or suboptimal.

CA-125 is one of the significant predictors for outcome of cytoreduction [16]. Other studies have suggested that the COP of CA $125>100 \mathrm{U} / \mathrm{ml}$ increases the occurrence of suboptimal cytoreduction [17]. Feng et al. stated that CA125 could predict optimal or suboptimal cytoreduction. A pre-operative COP of CA-125 $>500 \mathrm{U} / \mathrm{ml}$ had a sensitivity of $78 \%$ and specificity of $73 \%$ for predicting suboptimal cytoreduction [18]. Another study stated that $912 \mathrm{U} / \mathrm{ml}$ was an optimal cut-off value of CA-125 in predicting optimal and suboptimal cytoreduction [19]. The COP of serum CA125 levels still varies.

In our study, the COP of CA-125 was $222.5 \mathrm{U} / \mathrm{mL}$; based on the ROC method, the AUC value received was $77.5 \%$, suggesting that $77.5 \%$ of patients with serum CA-125 levels exceeded $222.5 \mathrm{U} / \mathrm{ml}$. This was predictive of suboptimal cytoreduction with $61.9 \%$ specificity, $75 \%$ sensitivity, and $69.8 \%$ accuracy. The specificity value of AKT2 was higher than that of CA-125, matching the current theory that pre-operative CA-125 serum level is not a specific tumor marker to diagnose ovarian cancer [3]. CA-125 also has a low specificity for malignant ovarian cancer because it often increases in patients with benign endometriosis [15].

When CA-125 and AKT2 were combined, the new COP was $410.45 \mathrm{U} / \mathrm{mL}$ with a specificity value of $61.9 \%$, sensitivity of $75 \%$, and the same accuracy value of $69.8 \%$. The AUC value obtained from the ROC method was $78.1 \%$, meaning that as many as $78.1 \%$ of patients with a combination of CA-125 and AKT2 value more than $410.45 \mathrm{U} / \mathrm{mL}$ may experience suboptimal cytoreduction. Based on the confidence interval, the combination of AKT2 and CA-125 value ranged from $65.9 \%$ to $90.3 \%$. Whether separate or in combination, AKT2 and CA-125 had the same accuracy level.

Currently, there is insufficient evidence to recommend the use of AKT levels in clinical practice as a predictor of epithelial ovarian cancer prognosis [15]. Further research will be necessary to substantiate our present findings. However, as the combination of AKT2 and CA-125 has a higher AUC value, it can be concluded that the combination of both tumor markers is beneficial than either used alone.

Finally, the success of cytoreductive surgery still depends on the experience of the surgeon as well as other factors such as FIGO cancer stage, histopathological type, and body mass index [19-21]. Findings from this research may prove useful in helping to predict outcomes following cytoreduction and patient prognosis.

The major limitation of our study was insufficient patient cohort. Therefore, future studies should involve a larger number of patients to increase the power of the study and the validity of our findings.

\section{Conclusions}

AKT2 and CA-125 serum levels are significantly different between the patients in suboptimal and optimal cytoreduction groups. AKT2 or CA-125 can be used as single tumor markers to predict cytoreduction, wherein the COP of AKT is $1.20 \mathrm{U} / \mathrm{mL}$ and the COP of CA-125 is $222.5 \mathrm{U} / \mathrm{mL}$. As both tumor markers are correlated, AKT2 and CA-125 can be used in combination to achieve a new COP of 410.45 $\mathrm{U} / \mathrm{mL}$, providing a better AUC value.

\section{Ethics Approval and Consent to Participate}

This study was approved by the Research Ethics Committee, Faculty of Medicine Padjadjaran University/Dr. Hasan Sadikin Hospital, Bandung, Indonesia, under trial registration number 36/UN6.KEP/EC/2018 on April 13 ${ }^{\text {th }}$, 2018. Suspected ovarian cancer patients were invited to 
participate in this study. Patients who were willing to become participants in this study were asked to fill and sign an informed consent form.

\section{Funding}

This study was supported by the Grants-in-Aid from the Ministry of Research, Technology and Higher Education of the Republic of Indonesia 06/E/KPT/2019(INSINAS).

\section{Authors' Contributions}

YMH, GNAW, and SRK designed the research study. YMH, GNAW, AK performed the research. MDLT, KIM, SRK provided help and advice on the experiments. YMH, MDLT, KIM analyzed the data. YMH, KIM, AK, SRK wrote the manuscript. All authors contributed to editorial changes in the manuscript. All authors read and approved the final manuscript.

\section{Availability of data and materials section}

The authors declare that the data will not be shared due to issues of patient confidentiality.

\section{Consent to Publish}

All authors declare that consent was given for publication of this study and accompanying images to be published.

\section{Acknowledgments}

Author(s) would like to acknowledge Wulan Ardhana Iswari, M.D. and Tendi Robby Setia, M.D. with appreciation for technical assistance.

\section{Conflict of interest}

Authors have declared that no competing interests exist.

Submitted: December 27, 2019

Accepted: June 17, 2019

Published: October 15, 2020

\section{References}

[1] WHO. GLOBOCAN (2012): Estimated Cancer Incidence, Mortality, and Prevalence Worldwide in 2012 v1.0 [Available from: http://globocan.iarc.fr/Pages/fact_sheets_population.aspx].

[2] Bray F., Ferlay J., Soerjomataram I., Siegel R.L., Torre L.A. and Jemal A.: "Global cancer statistics 2018: GLOBOCAN estimates of incidence and mortality worldwide for 36 cancers in 185 countries". Ca. Cancer J. Clin., 2018, 68, 394-424.

[3] Berek J.S., Friedlander M.L., Bast R.C.: "Epithelial Ovarian, Fallopian Tube, and Peritoneal Cancer". Holland-Frei Cancer Medicine, 2017, 65, 1-27.

[4] Berek J.S.: “Ovarian Cancer”. Hacker \&Amp;Amp Moore’s Essentials of Obstetrics and Gynecology, 2010, 412-419.

[5] Morgan R.J., Armstrong D.K., Alvarez R.D., Bakkum-Gamez J.N., Behbakht K., Chen L-M, et al. NCCN Clinical Practice Guidelines in Oncology (NCCN Guidelines $\left.{ }^{\circledR}\right)$. Ovarian cancer, including fallopian tube cancer and primary peritoneal cancer: NCCN.org 2015 [Available from: http://www.nccn.org/professionals/physician_gls/ pdf/ovarian.pdf].
[6] Prat J. The International Federation of Gynecologists and Obstetricians. New FIGO ovarian cancer staging guidelines 2014 [Available from: https://www.sgo.org/clinical-practice/guidelines/ new-figo-ovarian-cancer-staging-guidelines/].

[7] Saitou M., Iida Y., Komazaki H., Narui C., Matsuno K., Kawabata A., et al.: "Success rate and safety of tumor debulking with diaphragmatic surgery for advanced epithelial ovarian cancer and peritoneal cancer". Arch. Gynecol. Obstet., 2015, 291, 641-646.

[8] Arab M., Jamdar F., Sadat Hosseini M., Ghodssi- Ghasemabadi R., Farzaneh F., Ashrafganjoei T.: "Model for Prediction of Optimal Debulking of Epithelial Ovarian Cancer". Asian Pacific Journal of Cancer Prevention: APJCP, 2018, 191319-1324.

[9] Suidan R.S., Ramirez P.T., Sarasohn D.M., Teitcher J.B., Mironov S., Iyer R.B., et al.: "A multicenter prospective trial evaluating the ability of preoperative computed tomography scan and serum CA-125 to predict suboptimal cytoreduction at primary debulking surgery for advanced ovarian, fallopian tube, and peritoneal cancer". Gynecol. Oncol., 2014, 134, 455-461.

[10] Comamala M., Pinard M., Thériault C., Matte I., Albert A., Boivin M., et al.: "Downregulation of cell surface CA125/MUC16 induces epithelial-to-mesenchymal transition and restores EGFR signalling in NIH:OVCAR3 ovarian carcinoma cells". Br. J. Cancer, 2011, 104, 989-999.

[11] Barron C.C., Bilan P.J., Tsakiridis T., Tsiani E.: "Facilitative glucose transporters: Implications for cancer detection, prognosis and treatment". Metabolism, 2016, 65, 124-139.

[12] Menczer J., Ben-Shem E., Golan A., Levy T.: “The Significance of Normal Pretreatment Levels of CA125 $(<35 \mathrm{U} / \mathrm{mL})$ in Epithelial Ovarian Carcinoma". Rambam Maimonides Medical Journal, 2015, 6, e0005.

[13] Zheng B., Geng L., Zeng L., Liu F., Huang Q.: “AKT2 contributes to increase ovarian cancer cell migration and invasion through the AKT2-PKM2-STAT3NF-кB axis". Cell. Signal., 2018, 45, 122-131.

[14] Linnerth-Petrik N.M., Santry L. A., Moorehead R., Jücker M., Wootton S.K., Petrik J.: "Akt isoform specific effects in ovarian cancer progression". Oncotarget, 2016, 7, 74820-74833.

[15] Cai J., Xu L., Tang H., Yang Q., Yi X., Fang Y., et al.: “The Role of the PTEN/PI3K/Akt Pathway on Prognosis in Epithelial Ovarian Cancer: A Meta-Analysis". the Oncologist, 2014, 19, 528-535.

[16] Sehouli J., Grabowski J.P.: "Surgery for recurrent ovarian cancer: Options and limits". Best Practice \& Research Clinical Obstetrics \& Gynaecology, 2017, 41, 88-95.

[17] Chesnais M., Lecuru F., Mimouni M., Ngo C., Fauconnier A., Huchon $\mathrm{C}$.: "A pre-operative predictive score to evaluate the feasibility of complete cytoreductive surgery in patients with epithelial ovarian cancer". Plos one, 2017, 12, e0187245.

[18] Feng L., Liao S., Li L.: "Preoperative serum levels of HE4 and CA125 predict primary optimal cytoreduction in advanced epithelial ovarian cancer: a preliminary model study". J. Ovarian Res., 2020,13 .

[19] Zeng J., Yin J., Song X., Jin Y., Li Y., Pan L.: "Reduction of CA125 Levels During Neoadjuvant Chemotherapy Can Predict Cytoreduction to No Visible Residual Disease in Patients with Advanced Epithelial Ovarian Cancer, Primary Carcinoma of Fallopian tube and Peritoneal Carcinoma". Journal of Cancer, 2016, 7, 2327-2332.

[20] Kim S.J., Rosen B., Fan I., Ivanova A., McLaughlin J. R., Risch H., et al.: "Epidemiologic factors that predict long-term survival following a diagnosis of epithelial ovarian cancer". Br. J. Cancer, 2017, 116, 964-971.

[21] Purcell S.A., Elliott S.A., Kroenke C.H., Sawyer M.B., Prado C.M.: "Impact of Body Weight and Body Composition on Ovarian Cancer Prognosis". Curr. Oncol. Rep., 2016, 18, 8.

Corresponding Author:

KEMALA ISNAINIASIH MANTILIDEWI, MD., PhD

Department of Obstetrics and Gynecology, Faculty of Medicine, Universitas Padjadjaran-Dr. Hasan Sadikin General Hospital, Jl. Pasteur No. 38, Bandung 40161, West Java, (Indonesia) e-mail: kmantilidewi@gmail.com 